\title{
Deterioration Diagnosis Method for Ball Bearings that Continue Minute Swaying Motion*
}

\author{
Daisuke MATSUKA** and Mikio TOKUYAMA** \\ ${ }^{* *}$ Hitachi, Ltd. \\ 292, Yoshida, Totsuka, Yokohama, 244-0817, Japan \\ E-mail: daisuke.matsuka.ea@hitachi.com
}

\begin{abstract}
The galvanometer scanner is a type of swaying motor used in laser-drilling machines that is able to position the laser at more than 2,000 points per second. In recent years, the number of holes in printed circuit boards for mobile phones and other electronic devices has increased, so printed circuit boards now have layers and high-density patterns. Therefore, most of the motions of galvanometer scanners are for a short distance and time. The balls in the bearings do not go into $360^{\circ}$ rolls, so fretting damage occurs over a long period of time. This is likely to have an adverse effect on positioning accuracy.

In this paper, we propose a deterioration diagnosis method for galvanometer scanners that focuses on ball bearing wear. The proposed method can be used to determine the extent of damage and if the ball bearings need to be replaced. The effectiveness of the proposed method was verified by experiments using galvanometer scanners with laser drilling machines over a long period of time.
\end{abstract}

Key words: Ball Bearing, Fretting Damage, Positioning Accuracy, Motion Control

\section{Introduction}

The galvanometer scanner is a type of swaying motor used in laser-drilling machines that is able to position the laser at more than 2,000 holes per second ${ }^{(1)-(3)}$. These holes are used for the electrical connections of printed wiring boards (PWBs) produced layer-by-layer. In recent years, the number of holes in printed circuit boards for mobile phones and other electronic devices has increased ${ }^{(4)}$, so PWBs now have many layers and high-density patterns. Therefore, most of the motions of galvanometer scanners are for a short distance and time. The balls in the ball bearings do not go into $360^{\circ}$ rolls, so fretting damage occurs over a long period of time. This is likely to have an adverse effect on positioning accuracy.

For predicting the life of ball bearings' rotating motion, ISO 281 specifies methods of calculating the basic rating life of a ball bearing, which is the life associated with $90 \%$ reliability ${ }^{(5)}$. Another formula for calculating the basic rating life of a ball bearing for swaying motion has also been proposed ${ }^{(6)}$; however, galvanometer scanners usually move small angle of less than $2^{\circ}$, and their bearings do not rotate, so those methods cannot be applied for galvanometer scanners.

One way of determining bearing wear is checking the vibration and sound caused by the rotating motion of the ball bearing ${ }^{(7)}$. The relation between swaying angle and degree of fretting wear had been investigated by way of experiment ${ }^{(8)}$. For positioning accuracy, a mathematical model of the feed drive system that can evaluate the effect of ball screw and

${ }^{*}$ Received 11 June, 2012 (№. 12-0257) [DOI: 10.1299/jamdsm.7.30]

Copyright () 2013 by JSME 
support bearings wear has also previously been proposed ${ }^{(9)}$.

In this study, we propose a deterioration diagnosis method for galvanometer scanners that focuses on ball bearing wear. The proposed method can be used to determine the extent of damage that has a negative effect on positioning accuracy without having to disassemble equipment. The effectiveness of the proposed method was verified by experiments using galvanometer scanners with laser drilling machines over a long period of time.

\section{Positioning system of mirrors}

Figure 1 shows a structural diagram of the positioning system for the laser drilling machines. These machines are used in a computerized numerically controlled (CNC) hierarchical system, and the supervisor controller sends target angle commands to the galvano controller based on CAM data of the PCBs. The galvanometer scanner is operated by the current controlled amplifier. Figure 2 shows a cross-section of the galvanometer scanner. The shaft component, which consists of the coil, mirror, and scale for the angular sensor, is held by two ball bearings. The rotating angle of the shaft is measured by angular sensor and the sensor is used for the positioning servo control. A permanent magnet is located on the stator. Its axis of magnetization is in the radial direction, and the magnetic field is caused by the permanent magnet and inner yoke. The magnetic flux exists at air gap. When the coil carries a current, force is generated by the magnetic flux density and the current in a circumferential direction. The coils are attached to the shaft; therefore, the force is the torque of the galvanometer scanner. The torque is proportional to the strength of the current.

The frequency response of the positioning system of the galvanometer scanner used in this study is indicated by the solid line in Fig. 3. The mathematical model $P_{s}(s)$ is given as

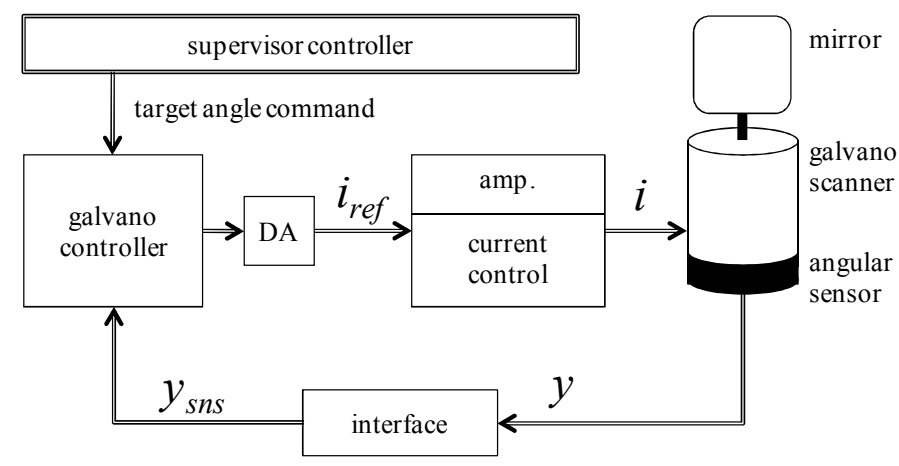

Fig. 1 Structural diagram of positioning system for laser drilling machines.

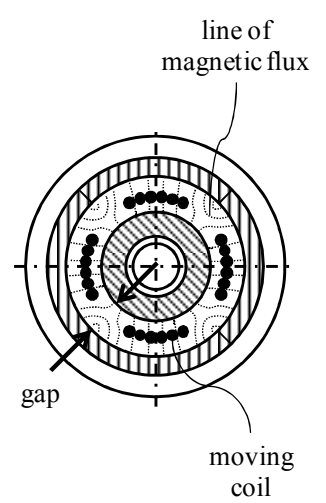

(a) Radial direction

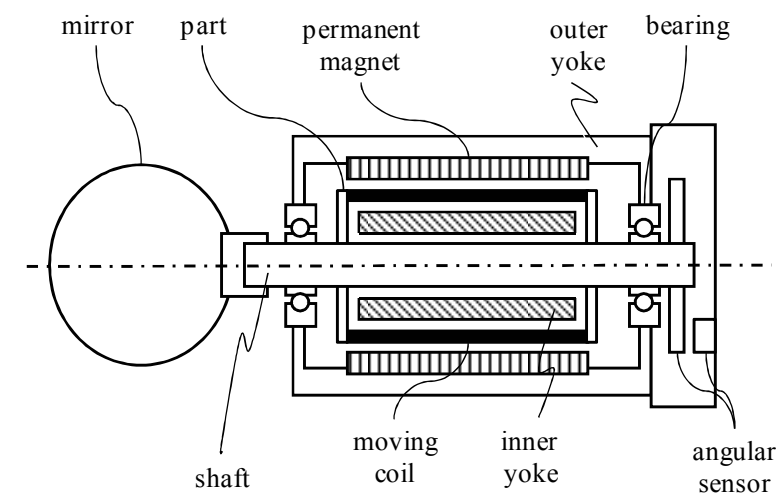

(b) Longer direction

Fig. 2 Cross-section of galvanometer scanner. 


$$
\begin{aligned}
P_{s}(s) & =\frac{y_{\text {sns }}(s)}{i_{\text {ref }}(s)} \\
& =e^{-L S} K_{p}\left(\sum_{i=1}^{l} \frac{k_{i}}{s^{2}+2 \zeta_{i} \omega_{i} s+\omega_{i}^{2}}\right)
\end{aligned}
$$

where $l$ is the number of modes under consideration, $k$ is the residue of each mode, $\omega$ and $\zeta$ are the natural frequency $(\mathrm{rad} / \mathrm{s})$ and damping ratios of the resonances, respectively, $L$ is the corresponding time delay, and $K_{p}$ is the plant gain. These parameters are fixed so that the model's frequency response coincides with the measured frequency response (shown by the solid line in Fig. 3). The broken line in Fig. 3 represents the frequency response given by this mathematical model, in which $l$ is set at four, and the values of the other parameter are as listed in Table 1.
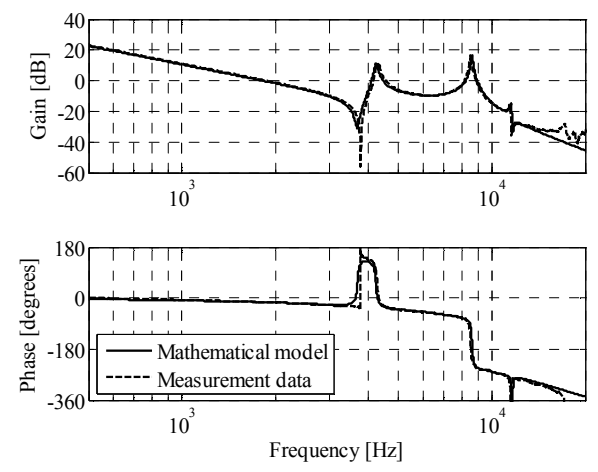

Fig. 3 Bode plot of the controlled object.

Table 1 Parameters of controlled object.

\begin{tabular}{cccc}
\hline$l$ & $\omega_{i}(\mathrm{rad} / \mathrm{s})$ & $\zeta_{i}$ & $k_{i}$ \\
\hline 1 & 0 & 0 & 1 \\
\hline 2 & $2 \pi \times 4200$ & 0.01 & 0.44 \\
\hline 3 & $2 \pi \times 8600$ & 0.01 & -1.83 \\
\hline 4 & $2 \pi \times 11600$ & 0.01 & 0.05 \\
\hline
\end{tabular}

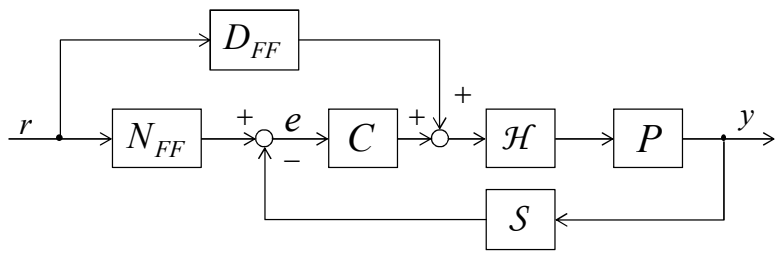

Fig. 4 Block diagram of positioning system.

The positioning system of the galvanometer scanner uses a two-degrees-of-freedom control framework. Figure 4 shows a block diagram of the positioning system, where $N_{F F}$ and $D_{F F}$ are feedforward compensators using a coprime factorization approach, $C$ is the feedback compensator, $P$ is the controlled object, $\mathcal{H}$ is the sample holder, $S$ is the sampler, $r$ is the position input, $y$ is the angular signal, and $e$ is the positioning error. It is too difficult to obtain the angle of mirror movement at the galvanometer scanner, so the positioning system uses an angular sensor for feedback control. Thus, the positioning system is a semi-closed loop framework. The feedback compensator consists of an integral compensator and state observer. Parameters of the feedback compensator are set to enlarge the phase margin to more than $25^{\circ}$ and the gain margin to more than $5.0 \mathrm{~dB}$. 


\section{Deterioration diagnosis method for ball bearings}

\subsection{Overview of the proposed method}

The characteristics of a galvanometer scanner that has been used for a long time are changed by many factors. Among these is fretting damage, which is caused by continuous minute reciprocating swaying motions that has an adverse effect on positioning accuracy and is fatal for the laser drilling machine due to inevitable operational errors. This clearly demonstrates the need for a deterioration diagnosis method for galvanometer scanners. Previously, galvanometer scanners tended to be checked by hand after the scanners broke down or processed holes poorly, and thus the deterioration of scanners and its effect on positioning accuracy have not evaluated from a quantitative standpoint.

In this study, the proposed method focuses on the connection between torque variation and deterioration of ball bearings and is able to evaluate deterioration of ball bearings quantitatively ${ }^{(10)(11)}$. There are many reasons about changed torque variation, for example, irreversible thermal demagnetized. The torque proportions of those galvanometer scanners change around the movement range. On the other hand, the galvanometer scanner, whose bearings have heavy fretting damage, has rolling resistances at some positions in the movement range. This trend is different from demagnetized. Therefore, if we can obtain the information where and how big the torque variation occurs, we can separate the factors of the torque variation. Also we can make deterioration assessment about ball bearings.

Figure 5 shows a flow chart of the proposed method as it diagnoses ball bearing deterioration. This process consists of two parts: 1) measuring torque proportion (steps 10-40 in Fig. 5), and 2) calculating torque variation and determining the deterioration of ball bearings. How these steps unfold is described in detail in the following sections.

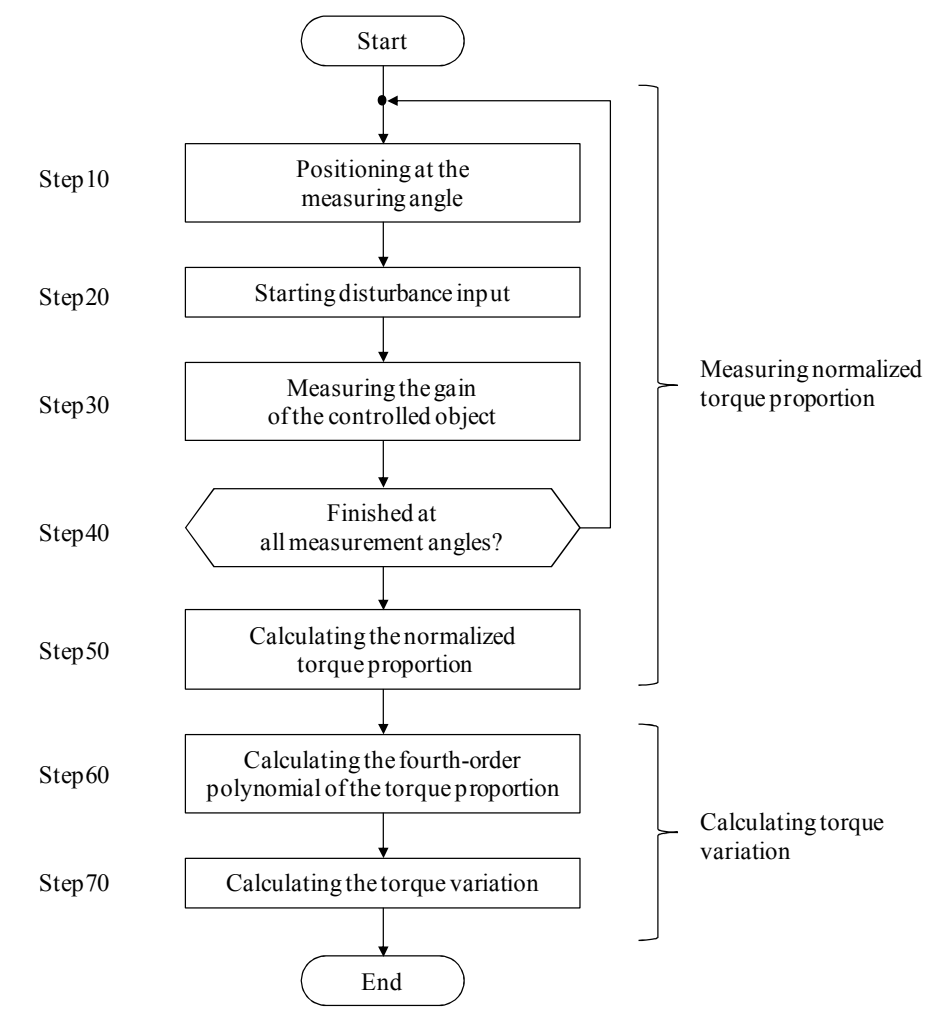

Fig. 5 Flow chart of proposed method as it diagnoses ball bearing deterioration.

\subsection{Measuring normalized torque proportion}

Figure 6 shows the block diagram for measuring normalized torque proportion, where $C, P, \mathcal{H}, S, r$, and $y$ are same as in Fig. $4, d$ is the disturbance input which is the sine wave, 
and $H(z)$ are same band-pass filters. The band-pass filter $H(z)$ is consisted of second-order Butterworth low-pass filters $H_{l p f}(z)$ and high-pass filter $H_{h p,}(z)$ which are serial connection and is given as

$$
H(z)=H_{l p f}(z) \cdot H_{l p f}(z) \cdot H_{h p f}(z) \cdot H_{h p f}(z) .
$$

The cutoff frequency of $H_{l p f}(z)$ and $H_{h p \rho}(z)$ are set at $1 \mathrm{kHz}$. Those filters are made by bilinear Z-transform.

The first step of the sequence is step 10 (shown in Fig. 5), which is the positioning at the measuring angle $\theta_{m}(m=1,2, \ldots, i, \ldots 1000)$. There are 1,000 measuring angles at equal space in the movement range, $\pm 30 \mathrm{rad} / \mathrm{unit}$. When the galvanometer is finished positioning at $\theta_{i}$, we start inputting the disturbance (step 20). The controlled object has some mechanical resonance; therefore, the frequency of the input disturbance is selected as 1 $\mathrm{kHz}$, because the frequency area between $0.5 \mathrm{kHz}$ to $2 \mathrm{kHz}$ is low effect of the resonance. The angular sensor of the galvanometer scanner uses the interpolatory algorithm between the optical grids for high resolution, so the amplitude of input disturbance is adjusted to angular signal to $0.67 \mathrm{rad} / \mathrm{unit}$ which is larger than the optical grid interval of the angular sensor, $0.33 \mathrm{rad} / \mathrm{unit}$, for reducing interpolated error of the angular sensor. This sine wave is able to select the direct-current (DC) input for the input disturbance, although using a sine wave is better than using a DC input because it can reduce angular sensor errors.

After the galvanometer's response is under steady state condition, we start obtaining $u_{f}(z)$ and $y_{f}(z)$. They are given as

$$
\begin{aligned}
& u_{f}(z)=H(z) u(z) \\
& y_{f}(z)=H(z) y(z) .
\end{aligned}
$$

And we get the gain $g_{i}$ of the controlled object at the measuring angle $\theta_{i}$ (step 30) and are given as

$$
\begin{aligned}
& g_{i}=\left.\frac{u_{\text {peak }}}{y_{\text {peak }}}\right|_{\theta_{i}} \\
& u_{\text {peak }}=\sup a b s\left(u_{f}(z)\right) . \\
& y_{\text {peak }}=\sup a b s\left(y_{f}(z)\right)
\end{aligned}
$$

After we measure the gain of the controlled object at all measuring angle, we can get the normalized torque proportion $L_{i}$ (step 50) and given as

$$
L_{i}=\frac{g_{i}}{g_{c}}
$$

where $g_{c}$ is the gain of the controlled object at the center of the movement range. Steps $10-50$ are the sequence for measuring normalized torque proportion. The amplitude of the disturbance input is smaller than the control input at the normal process, so it does not affect the machine in an adverse way.

Figure 7 shows the normalized torque proportion of the galvanometer scanner. The angle of the measurement position is normalized by the basic movement angle, and we used $\mathrm{rad} / \mathrm{unit}$ for the units. The torque proportion of the galvanometer scanner is not flat because of the non-uniform magnetic flux density in the range of movement. 


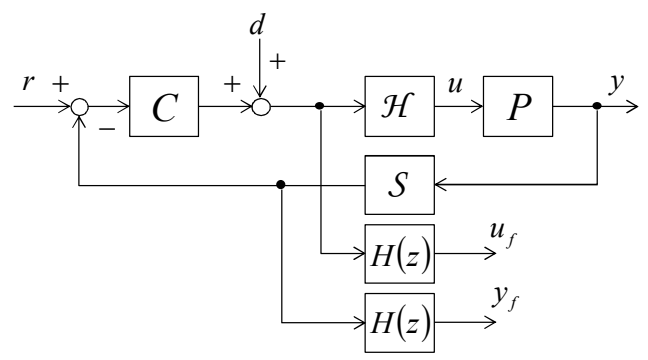

Fig. 6 Block diagram of measuring system.

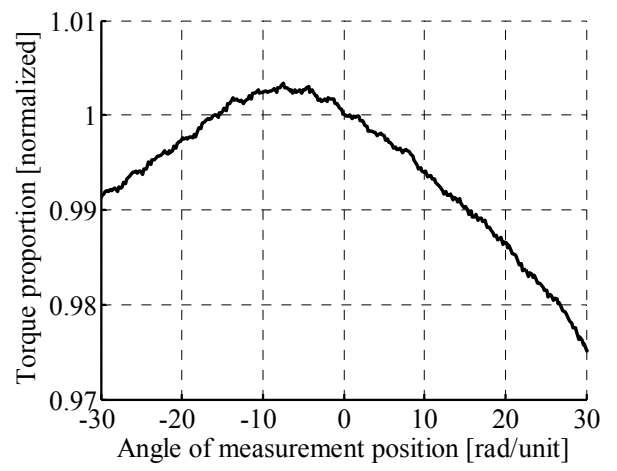

Fig. 7 Normalized torque proportion of normal galvanometer scanner.

\subsection{Deterioration diagnosis method for ball bearings}

We expect the torque proportion to change if the ball bearings have been damaged, and we are thus able to determine bearing wear by examining the torque proportion of the galvanometer scanner. The torque proportion of the galvanometer scanner is convex upward because of the non-uniform magnetic flux density in the range of movement (Fig. 7), so we subtract the effect of non-uniform magnetic flux density from the torque proportion that was measured. We then used the result, torque variation, as the index of bearing wear. Various factors are suspected of causing torque variation, although knowledge obtained in the field indicates that the torque variation at the center and edge of movement is caused by bearing wear. The torque proportion $K_{c}$ caused by magnetic flux density is approximated by the fourth-order polynomial of angle $\theta$ and is given as

$$
K_{c} \approx a_{1} \theta^{4}+a_{2} \theta^{3}+a_{3} \theta^{2}+a_{4} \theta+a_{5},
$$

where $a_{1}$ through $a_{5}$ are calculated with the torque proportion and the coefficients are stored as memory. Figure 8 shows the torque variation of a galvanometer scanner that has been used for laser drilling machine in a little time. The torque variation is almost completely flat, which means the ball bearings are in good condition. Figure 9 shows the waveform of positioning error when the target angle was set to $1 \mathrm{rad} / \mathrm{unit}$ and repeated 100 times for positioning in a single direction. In Fig. 9, the bright line shows the 100th positioning error. This galvanometer scanner has a positioning error within about $4 \times 10^{-3} \mathrm{rad} / \mathrm{unit}$. The criterion for positioning accuracy is within $4 \times 10^{-3} \mathrm{rad} / \mathrm{unit}$, which means that this scanner falls within the guideline.

\section{Experiment}

To determine the effectiveness of the proposed method, we performed experiments with two galvanometer scanners that had been used for laser drilling machines over a long period of time. These scanners did not disassemble before the experiments had finished completely. 


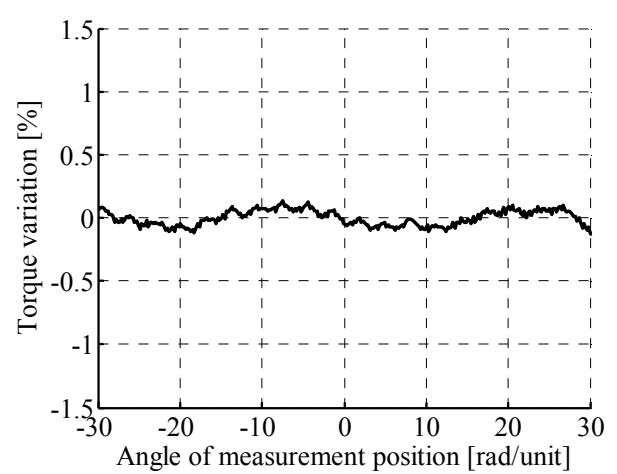

Fig. 8 Torque variation of normal galvanometer scanner.

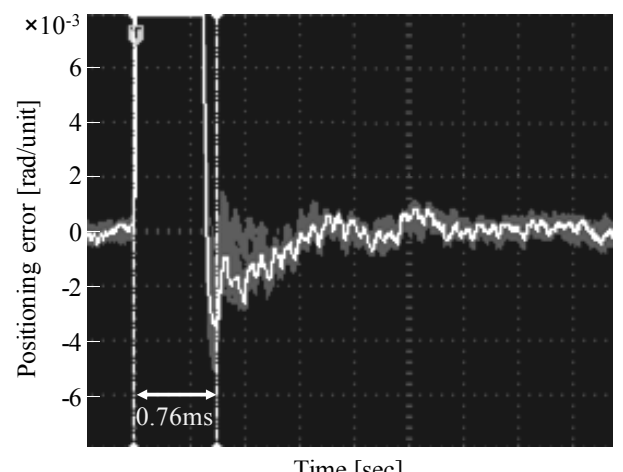

Time [sec]

Fig. 9 Waveform of positioning error of normal galvanometer scanner.

Scanner A had a little rolling resistance at the center of the movement range that was checked by hand-turning, while Scanner B had more rolling resistance at the center and both sides of the movement range. These characteristics are common among galvanometer scanners that have been pulled from the field due to deteriorating positioning accuracy. Figure 10 shows the torque variation calculated by the proposed method, and Table 2 lists the coefficients of the fourth-order polynomial of angle $\theta$ (as shown in Formula 6) at these scanners. As shown in Fig. 10, the torque variation of Scanner A is almost completely flat except at the center of its movement range, whereas Scanner B had large fluctuations at the center and sides of its movement range. The torque variation of Scanner B was over $1 \%$, so it is estimated that this scanner has ball bearings that are heavily damaged.

To see the effect of the bearing wear, we performed experiments on positioning control, in which the target angle was set to $1 \mathrm{rad} /$ unit and repeated 100 times for positioning in a single direction. Figure 11 shows waveforms of positioning error at Scanners A and B. In Fig. 11, the bright line shows the 100th positioning error. Scanner A had a positioning error over $4 \times 10^{-3} \mathrm{rad} /$ unit of the target angle, while Scanner B had a fluctuation of more than $6 \times 10^{-3} \mathrm{rad} /$ unit around the target angle, especially when it reached the target angle. If galvanometer scanners like this one are used in a laser drilling machine, the processed holes are almost always of poor quality due to bad positioning accuracy. It is possible to propose that a method for improving the positioning accuracy of galvanometer scanners that have a torque variation of more than $1 \%$, but considering the position of obtaining advanced warning of bearing wear, it is better to advise users to just replace the bearings.

After the experiments were completed, the two scanners were disassembled to check bearing wear. Figure 12 shows the inner race of the ball bearings at Scanners A and B. The inner race at Scanner A had a more modest degree of fretting wear, while the inner race of Scanner B demonstrated heavy fretting damage caused by continuous reciprocating motion. This indicates that the proposed method is able to quantitatively determine the extent of bearing damage without disassembled equipment.

From a practical standpoint, we propose our method be used to warn users when torque 
variation exceeds a pre-set level. To see how well this works, we applied the method to 42 galvanometer scanners that had all been used for a long time, including 10 whose bearings were damaged and would likely have an adverse effect on positioning accuracy. We were able to determine the extent of bearing damage by using the proposed method and could correctly distinguish all galvanometer scanners that needed replacing.

Table 2 Coefficients of four-order polynomial at scanners.

\begin{tabular}{|c|c|c|}
\hline & Scanner A & Scanner B \\
\hline$a_{1}$ & $2.84 \times 10^{-5}$ & $6.57 \times 10^{-7}$ \\
\hline $\mathrm{a}_{2}$ & $5.16 \times 10^{-6}$ & $-1.43 \times 10^{-5}$ \\
\hline$a_{3}$ & $-1.51 \times 10^{-3}$ & $-1.06 \times 10^{-3}$ \\
\hline $\mathrm{a}_{4}$ & $-7.88 \times 10^{-4}$ & $2.71 \times 10^{-4}$ \\
\hline$a_{5}$ & 1.04 & 1.00 \\
\hline
\end{tabular}
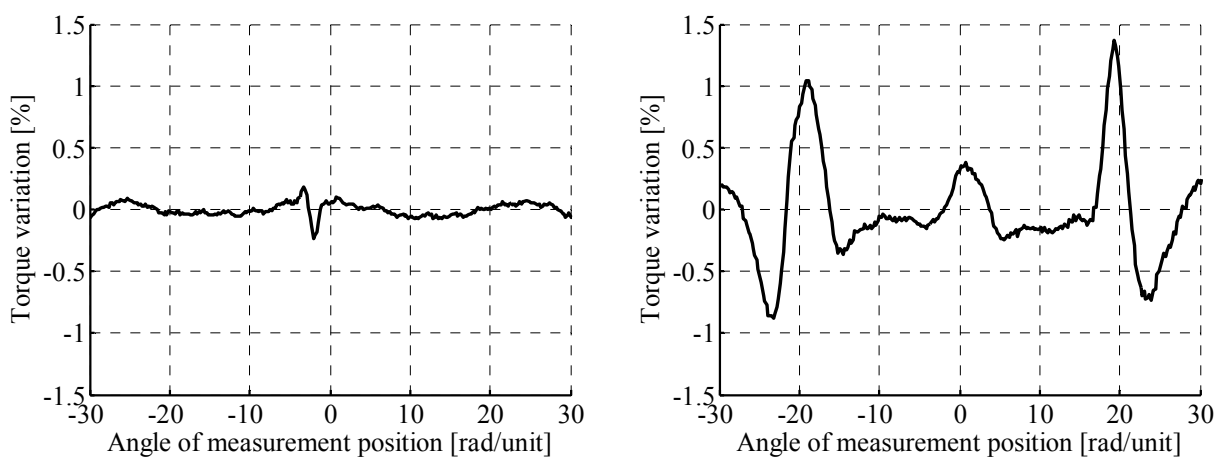

Fig. 10 Torque variations of Scanner A (left) and Scanner B (right).

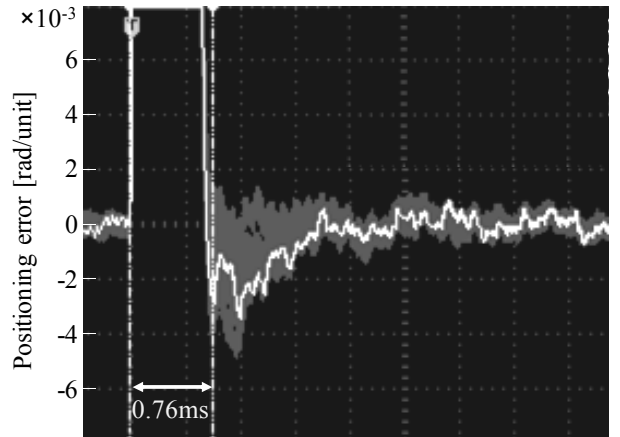

Time $[\mathrm{sec}]$

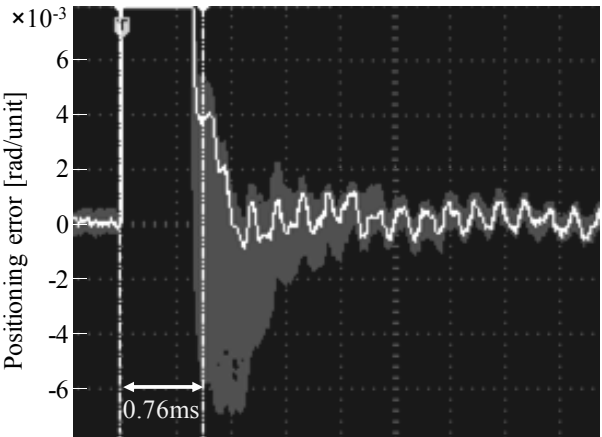

Time [sec]

Fig. 11 Waveform of positioning error at Scanner A (left) and Scanner B (right).
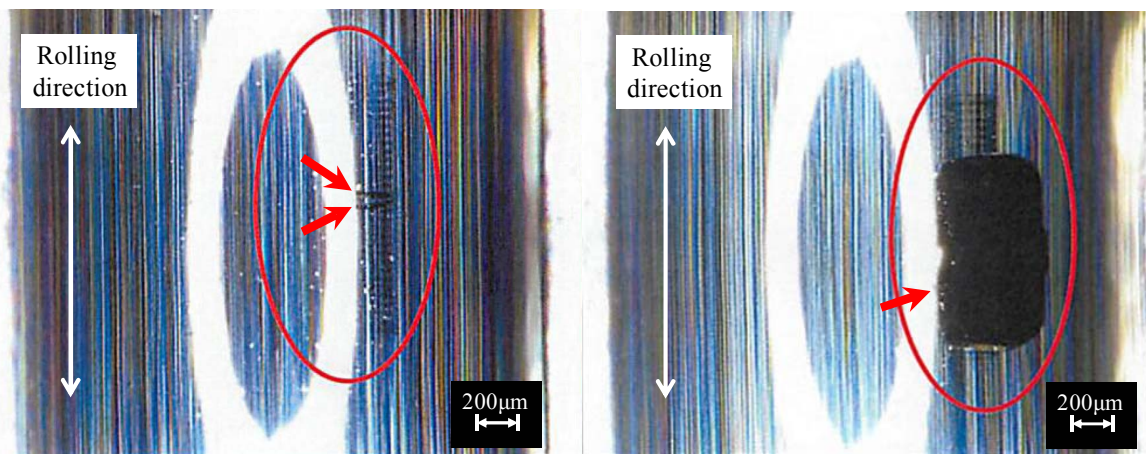

Fig. 12 Inner race of Scanner A (left) and Scanner B (right). 


\section{Conclusion}

We proposed a deterioration diagnosis method for the ball bearings of galvanometer scanners. The proposed method can be used to determine the extent of damage and if the ball bearings need to be replaced without having to disassemble any equipment. Results of an experiment using galvanometer scanners with laser drilling machines that had been used over a long period of time showed that the proposed method could effectively judge when it was necessary to replace the galvanometer scanner by examining the ball bearing wear.

\section{References}

(1) The Institute of Electrical Engineers of Japan, "The basics of latest laser processing and industrial application", Ohmsha, 2007.

(2) H. Otsuki, T. Ito, H. Aoyama, S. Tohyama, and H. Hirai, 2006, "Development of Laser Drilling Machine for Printed Wiring Boards", JSME Annual Meeting, 7: 331-332.

(3) N. Michigami, M. Yamaga, M. Kawamura, O. Kuze and S. Nakamura, "Manufacturing Device of Printed Circuit Board for High-density Multilayer Wiring", Hitachi Hyoron, Vol.93, No.02:192-197

(4) Japan Jisso Technology Roadmap Council, "Japan Jisso Technology Roadmap 2011", Japan Electronics and Information Technology Industrial association, 2011.

(5) ISO 281:2007, "Rolling bearing - Dynamic load ratings and rating life".

(6) K. Kimata, 1969, "Predicting the life of ball bearings' swaying motion", Bearing Engineer, 18, 2:2383-2391.

(7) E. Watabayashi, "Rolling bearing manual", Japan Standards Association, 1999.

(8) Q. Li, M. Shima, T. Yamamoto and J. Sato, 1995, "Study of Fretting Wear of Rolling Bearing (Part4) -Effects of Various Factors on Fretting Wear-", Journal of Japanese Society of Tribologists, 40, 12:1029-1036.

(9) R. Sato, 2011, "Mathematical Model of Feed Drive Systems with Wear of Ball Screw and Support bearing", Proceeding of the spring conference on the Japan society for Precision Engineering, 467-468.

(10) H. Otsuki, S. Toyama, K. Seki, Y. Okubo and D. Kitamura, US Patent 7,270,270 B1 (18, September, 2007).

(11) M. Tokuyama, D. Matsuka, H. Otsuki, S. Toyama and S. Fukushima, Japan Patent Kokai 2012-031314 (16, February, 2012) 